\title{
Chromatic bases for symmetric functions
}

\author{
Soojin Cho* \\ Department of Mathematics \\ Ajou University \\ Suwon 443-749, Korea \\ chosj@ajou.ac.kr
}

\author{
Stephanie van Willigenburg ${ }^{\dagger}$ \\ Department of Mathematics \\ University of British Columbia \\ Vancouver BC V6T 1Z2, Canada \\ steph@math.ubc.ca
}

Submitted: Sep 3, 2015; Accepted: Jan 5, 2016; Published: Jan 22, 2016

Mathematics Subject Classifications: 05E05, 05C15, 05C25

\begin{abstract}
In this note we obtain numerous new bases for the algebra of symmetric functions whose generators are chromatic symmetric functions. More precisely, if $\left\{G_{k}\right\}_{k \geqslant 1}$ is a set of connected graphs such that $G_{k}$ has $k$ vertices for each $k$, then the set of all chromatic symmetric functions $\left\{X_{G_{k}}\right\}_{k \geqslant 1}$ generates the algebra of symmetric functions. We also obtain explicit expressions for the generators arising from complete graphs, star graphs, path graphs and cycle graphs.
\end{abstract}

Keywords: chromatic symmetric function, complete graph, star graph, path, cycle

\section{Introduction}

In [7] Stanley defined a symmetric function $X_{G}$ that was reliant on a finite simple graph $G$, called the chromatic symmetric function of $G$. He proved that $X_{G}$ specializes to the chromatic polynomial of $G$ and generalizes other chromatic polynomial properties, although intriguingly not the deletion-contraction property. Since then the chromatic symmetric function $X_{G}$ has been the genesis of two long-standing conjectures in algebraic combinatorics. The first of these conjectures that the chromatic symmetric functions of all $(3+1)$-free posets are a positive linear combination of elementary symmetric functions, for which a variety of evidence exists $[3,4,8]$. The second of these conjectures that the chromatic symmetric function distinguishes non-isomorphic trees. This conjecture has been confirmed for spiders [5] plus a variety of caterpillars [1, 5], and towards a different approach a sufficient condition for graphs to have equal chromatic symmetric functions has also been discovered [6].

* Supported by Basic Science Research Program through the National Research Foundation of Korea (NRF) funded by the Ministry of Education (NRF-2015R1D1A1A01057476).

${ }^{\dagger}$ Supported in part by the National Sciences and Engineering Research Council of Canada. 
In this vein of a different perspective on the chromatic symmetric function we provide a potential new tool, namely we determine a myriad of new bases for the algebra of symmetric functions, whose generators are chromatic symmetric functions (Theorem 5) and give explicit expansions for the generators arising from well-known graphs (Theorem 8).

\section{Chromatic symmetric function bases}

We begin by recalling concepts that will be useful later, and by defining the algebra of symmetric functions. A partition $\lambda=\left(\lambda_{1}, \ldots, \lambda_{\ell}\right)$ of $n$, denoted by $\lambda \vdash n$, is a list of positive integers whose parts $\lambda_{i}$ satisfy $\lambda_{1} \geqslant \cdots \geqslant \lambda_{\ell}$ and $\sum_{i=1}^{\ell} \lambda_{i}=n$. If $\lambda$ has exactly $m_{i}$ parts equal to $i$ for $1 \leqslant i \leqslant n$ we will sometimes denote $\lambda$ by $\lambda=\left(1^{m_{1}}, \ldots, n^{m_{n}}\right)$. Also, given partitions of $n, \lambda=\left(\lambda_{1}, \ldots, \lambda_{\ell}\right)$ and $\mu=\left(\mu_{1}, \ldots, \mu_{m}\right)$ we say $\mu \leqslant \lambda$ in lexicographic order if $\mu=\lambda$ or $\mu_{i}=\lambda_{i}$ for $i<j$ and $\mu_{j}<\lambda_{j}$ for some $1 \leqslant j \leqslant m$.

The algebra of symmetric functions is a subalgebra of $\mathbb{Q}\left[\left[x_{1}, x_{2}, \ldots\right]\right]$ and can be defined as follows. We define the $i$-th power sum symmetric function $p_{i}, i \geqslant 1$ to be

$$
p_{i}=x_{1}^{i}+x_{2}^{i}+x_{3}^{i}+\cdots
$$

and given a partition $\lambda=\left(\lambda_{1}, \ldots, \lambda_{\ell}\right)$, we define the power sum symmetric function $p_{\lambda}$ to be

$$
p_{\lambda}=p_{\lambda_{1}} \cdots p_{\lambda_{\ell}}
$$

Then the algebra of symmetric functions, $\Lambda$, is the graded algebra

$$
\Lambda=\Lambda^{0} \oplus \Lambda^{1} \oplus \cdots
$$

where $\Lambda^{0}=\operatorname{span}\{1\}=\mathbb{Q}$ and for $n \geqslant 1$

$$
\Lambda^{n}=\operatorname{span}\left\{p_{\lambda} \mid \lambda \vdash n\right\} .
$$

The power sum symmetric functions in fact form a basis for $\Lambda$. Other well-known bases include the basis of Schur functions, the basis of complete homogeneous symmetric functions and the basis of elementary symmetric functions, whose $i$-th elementary symmetric function $e_{i}, i \geqslant 1$ is defined to be

$$
e_{i}=\sum_{j_{1}<\cdots<j_{i}} x_{j_{1}} \cdots x_{j_{i}}
$$

leading to the celebrated fundamental theorem of symmetric functions, which states that

$$
\Lambda=\mathbb{Q}\left[e_{1}, e_{2}, \ldots\right] .
$$

Our object of study is a further symmetric function, known as the chromatic symmetric function, which is reliant on a graph that is finite and simple, and from here onwards we will assume that all our graphs satisfy these properties. We are now almost ready to define the chromatic symmetric function of a graph, but before we do we recall the notion 
of a proper coloring. Given a graph $G$ with vertex set $V$ a proper coloring $\kappa$ of $G$ is a function

$$
\kappa: V \rightarrow\{1,2, \ldots\}
$$

such that if $v_{1}, v_{2} \in V$ are adjacent, then $\kappa\left(v_{1}\right) \neq \kappa\left(v_{2}\right)$.

Definition 1. For a graph $G$ with vertex set $V=\left\{v_{1}, \ldots, v_{n}\right\}$ and edge set $E$ the chromatic symmetric function of $G$ is defined to be

$$
X_{G}=\sum_{\kappa} x_{\kappa\left(v_{1}\right)} \cdots x_{\kappa\left(v_{n}\right)}
$$

where the sum is over all proper colorings $\kappa$ of $G$.

Given a graph $G$ with vertex set $V=\left\{v_{1}, \ldots, v_{n}\right\}$ and edge set $E$, and a subset $S \subseteq E$, let $\lambda(S)$ be the partition of $n$ whose parts are equal to the number of vertices in the connected components of the spanning subgraph of $G$ with vertex set $V$ and edge set $S$. We say a set partition $\pi=\left\{B_{1}, \ldots, B_{k}\right\}$ of $V$ is connected if the subgraph of $G$ determined by $B_{i}$ is connected for each $i$, and the lattice of contractions $L_{G}$ of $G$ is the set of all connected partitions of $V$ partially ordered by refinement so that the unique minimal element $\hat{0}$ of $L_{G}$ is the partition into $n$ one element blocks. Lastly, given $\pi=\left\{B_{1}, \ldots, B_{k}\right\} \in L_{G}$, the type of $\pi$, denoted by type $(\pi)$, is the partition obtained by rearranging $\left|B_{1}\right|, \ldots,\left|B_{k}\right|$ in weakly decreasing order. With this in mind we have the following.

Lemma 2. [7, Theorems 2.5 and 2.6] For a graph $G$ with vertex set $V$ and edge set $E$ we have that

1. $X_{G}=\sum_{S \subseteq E}(-1)^{|S|} p_{\lambda(S)}$,

2. $X_{G}=\sum_{\pi \in L_{G}} \mu(\hat{0}, \pi) p_{\text {type }(\pi)}$, where $\mu$ is the Möbius function of $L_{G}$, and $\mu(\hat{0}, \pi)$ is non-zero for all $\pi \in L_{G}$.

The chromatic symmetric function also satisfies the following useful property.

Lemma 3. [7, Proposition 2.3] If a graph $G$ is a disjoint union of subgraphs $G_{1}, \ldots, G_{\ell}$, then $X_{G}=\prod_{i=1}^{\ell} X_{G_{i}}$.

We now need one last definition before we can state our theorem.

Definition 4. Let $\left\{G_{k}\right\}_{k \geqslant 1}$ be a set of connected graphs such that $G_{k}$ has $k$ vertices for each $k$, and let $\lambda=\left(\lambda_{1}, \ldots, \lambda_{\ell}\right)$ be a partition. Then

$$
G_{\lambda}=G_{\lambda_{1}} \cup \cdots \cup G_{\lambda_{\ell}}
$$

that is, $G_{\lambda}$ is the graph whose connected components are $G_{\lambda_{1}}, \ldots, G_{\lambda_{\ell}}$.

We can now determine a plethora of new bases for $\Lambda$. 
Theorem 5. Let $\left\{G_{k}\right\}_{k \geqslant 1}$ be a set of connected graphs such that $G_{k}$ has $k$ vertices for each $k$. Then

$$
\left\{X_{G_{\lambda}} \mid \lambda \vdash n\right\}
$$

is a $\mathbb{Q}$-basis of $\Lambda^{n}$. Plus we have that

$$
\Lambda=\mathbb{Q}\left[X_{G_{1}}, X_{G_{2}}, \ldots\right]
$$

and the $X_{G_{k}}$ are algebraically independent over $\mathbb{Q}$.

Proof. Let $\lambda=\left(\lambda_{1}, \ldots, \lambda_{\ell}\right) \vdash n$ and $V_{i}$ be the sets of vertices in $G_{\lambda_{i}}$ for $1 \leqslant i \leqslant \ell$. Then

$$
V=\biguplus_{i=1}^{\ell} V_{i}
$$

is the set of vertices in $G_{\lambda}$. By the definition of $G_{\lambda}$, we know that if $\pi \in L_{G_{\lambda}}$, then type $(\pi) \leqslant \lambda$ in lexicographic order. Thus by Lemma 2 it follows that

$$
X_{G_{\lambda}}=\sum_{\mu \leqslant \lambda} c_{\lambda \mu} p_{\mu}
$$

and, moreover, that $c_{\lambda \lambda}=\mu\left(\hat{0}, \pi_{\lambda}\right) \neq 0$ where $\pi_{\lambda}=\left(V_{1}, \ldots, V_{\ell}\right)$ is the unique connected partition of $V$ satisfying type $\left(\pi_{\lambda}\right)=\lambda$. Hence, $\left\{X_{G_{\lambda}} \mid \lambda \vdash n\right\}$ is a $\mathbb{Q}$-basis of $\Lambda^{n}$.

Since for $\lambda=\left(\lambda_{1}, \ldots, \lambda_{\ell}\right)$ we have

$$
X_{G_{\lambda}}=\prod_{i=1}^{\ell} X_{G_{\lambda_{i}}}
$$

by Lemma 3 and $\left\{X_{G_{\lambda}}\right\}_{\lambda \vdash n \geqslant 1} \cup\{1\}$ forms a $\mathbb{Q}$-basis for $\Lambda$, every element of $\Lambda$ is expressible uniquely as a polynomial in the $X_{G_{k}}$ and hence $\Lambda=\mathbb{Q}\left[X_{G_{1}}, X_{G_{2}}, \ldots\right]$ and the $X_{G_{k}}$ are algebraically independent over $\mathbb{Q}$.

Example 6. If

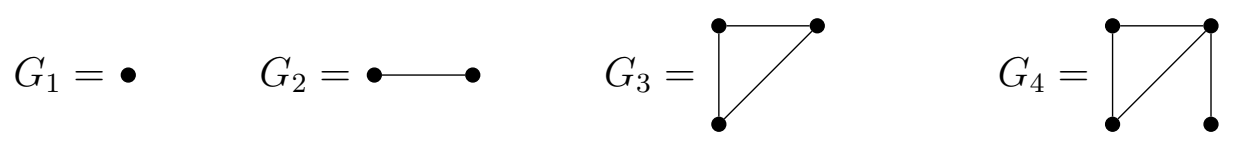

then $\left\{X_{G_{1}}, X_{G_{2}}, X_{G_{3}}, X_{G_{4}}\right\}$ is a set of generators for $\Lambda^{4}$ and

$$
\begin{aligned}
& X_{G_{(4)}}=X_{G_{4}}=-2 p_{(4)}+4 p_{(3,1)}+p_{(2,2)}-4 p_{(2,1,1)}+p_{(1,1,1,1)}, \\
& X_{G_{(3,1)}}=X_{G_{3}} X_{G_{1}}=\left(2 p_{(3)}-3 p_{(2,1)}+p_{(1,1,1)}\right) p_{(1)}=2 p_{(3,1)}-3 p_{(2,1,1)}+p_{(1,1,1,1)}, \\
& X_{G_{(2,2)}}=X_{G_{2}} X_{G_{2}}=\left(-p_{(2)}+p_{(1,1)}\right)^{2}=p_{(2,2)}-2 p_{(2,1,1)}+p_{(1,1,1,1)}, \\
& X_{G_{(2,1,1)}}=X_{G_{2}} X_{G_{1}} X_{G_{1}}=\left(-p_{(2)}+p_{(1,1)}\right) p_{(1)} p_{(1)}=-p_{(2,1,1)}+p_{(1,1,1,1)}, \text { and } \\
& X_{G_{(1,1,1,1)}}=X_{G_{1}} X_{G_{1}} X_{G_{1}} X_{G_{1}}=p_{(1)}^{4}=p_{(1,1,1,1)}
\end{aligned}
$$

is a $\mathbb{Q}$-basis of $\Lambda^{4}$.

Alternatively, if $A, B, C, D$ are as below, then $\left\{X_{A}, X_{B}, X_{C}, X_{D}\right\}$ is a set of generators for $\Lambda^{4}$ and

$$
\left\{X_{D}, X_{C} X_{A}, X_{B} X_{B}, X_{B} X_{A} X_{A}, X_{A} X_{A} X_{A} X_{A}\right\}
$$

is a $\mathbb{Q}$-basis of $\Lambda^{4}$. 


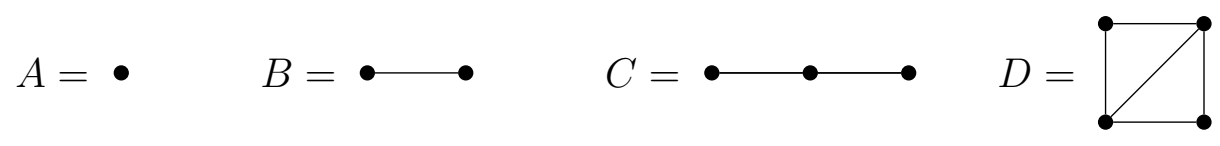

Remark 7. Observe that the only connected graph on two vertices is $G_{2}$ above, and

$$
e_{2}=\sum_{j_{1}<j_{2}} x_{j_{1}} x_{j_{2}}=\frac{1}{2} X_{G_{2}}
$$

Therefore, while every $\left\{X_{G_{\lambda}}\right\}_{\lambda \vdash n \geqslant 1} \cup\{1\}$ is a $\mathbb{Q}$-basis of $\Lambda$ it is never a $\mathbb{Z}$-basis of $\Lambda$.

\section{Chromatic symmetric functions for classes of graphs}

In this section, we compute chromatic symmetric functions for some particular connected graphs, whose definitions we include for clarity. The complete graph $K_{n}, n \geqslant 1$ has $n$ vertices each pair of which are adjacent. The star graph $S_{n+1},(n+1) \geqslant 1$ has $n+1$ vertices and is the tree with one vertex of degree $n$ and $n$ vertices of degree one. The path graph $P_{n}, n \geqslant 1$ has $n$ vertices and is the tree with 2 vertices of degree one and $n-2$ vertices of degree 2 for $n \geqslant 2$ and $P_{1}=K_{1}$. Lastly, the cycle graph $C_{n}, n \geqslant 1$ is the connected graph with $n$ vertices of degree 2 for $n \geqslant 3, C_{2}=K_{2}, C_{1}=K_{1}$. The chromatic symmetric functions of Ferrers graphs, naturally related to $\Lambda$ via Ferrers diagrams, were computed in [2]. We note that the third formula appears in the second proof of [7, Proposition 5.3] that gives the generating function for $X_{P_{n}}$, and the generating function for $X_{C_{n}}$ is given in [7, Proposition 5.4].

Theorem 8. 1. If $K_{n}$ is the complete graph with $n \geqslant 1$ vertices, then

$$
X_{K_{n}}=n ! e_{n}
$$

2. If $S_{n+1}$ is the star graph with $(n+1) \geqslant 1$ vertices, then

$$
X_{S_{n+1}}=\sum_{r=0}^{n}(-1)^{r}\left(\begin{array}{l}
n \\
r
\end{array}\right) p_{\left(r+1,1^{n-r}\right)}
$$

3. If $P_{n}$ is the path graph with $n \geqslant 1$ vertices, then

$$
X_{P_{n}}=\sum_{\lambda=\left(1^{\left.m_{1}, \ldots, n^{m_{n}}\right) \vdash n}\right.}(-1)^{n-\sum_{i=1}^{n} m_{i}} \frac{\left(\sum_{i=1}^{n} m_{i}\right) !}{\prod_{i=1}^{n}\left(m_{i}\right) !} p_{\lambda} .
$$

4. If $C_{n}$ is the cycle graph with $n \geqslant 1$ vertices, then

$$
X_{C_{n}}=\sum_{\lambda=\left(1^{\left.m_{1}, \ldots, n^{m_{n}}\right) \vdash n}\right.}(-1)^{n-\sum_{i=1}^{n} m_{i}} \frac{\left(\sum_{i=1}^{n} m_{i}\right) !}{\prod_{i=1}^{n}\left(m_{i}\right) !}\left(1+\sum_{j=2}^{n}(j-1) \frac{m_{j}}{\sum_{i=1}^{n} m_{i}}\right) p_{\lambda} .
$$


Proof. For $K_{n}$, since every vertex must be colored a different color and this can be done in $n$ ! ways, $X_{K_{n}}=n ! \sum_{j_{1}<\cdots<j_{n}} x_{j_{1}} \cdots x_{j_{n}}=n ! e_{n}$.

We now use the first part of Lemma 2 , which states that $X_{G}=\sum_{S \subseteq E}(-1)^{|S|} p_{\lambda(S)}$, for the remainder of the proof, where $E$ is the set of edges of our graph $G$.

For $S_{n+1}$, if we choose any $r$ edges, then $(r+1)$ vertices will make a connected component and the remaining $(n-r)$ vertices will be isolated. Hence the second part of the theorem follows.

For $P_{n}$, draw this graph on a horizontal axis. Now consider the spanning subgraph of $P_{n}$ with $n$ vertices and edge set $S \subseteq E, P_{n}^{S}$. Counting the number of vertices in each connected component of $P_{n}^{S}$ from left to right yields a list of positive integers when rearranged into weakly decreasing order yield a partition, say $\lambda=\left(1^{m_{1}}, \ldots, n^{m_{n}}\right) \vdash n$. Since the number of edge sets, $S$, that will yield $\lambda$ is $\frac{\left(\sum_{i=1}^{n} m_{i}\right) !}{\prod_{i=1}^{n}\left(m_{i}\right) !}$, and $|S|=\sum_{i=1}^{n}(i-1) m_{i}=$ $n-\sum_{i=1}^{n} m_{i}$ for such $S$, the third part is now proved.

For $C_{n}$ and a partition $\lambda=\left(1^{m_{1}}, \ldots, n^{m_{n}}\right) \vdash n$, we look for all subsets $S$ of the edge set $E$ that contribute to $p_{\lambda}$ in the expansion of $X_{C_{n}}$; that is, subsets $S$ satisfying $\lambda(S)=\lambda$. To this end, label the vertices of $C_{n}$ with $v_{1}, \ldots, v_{n}$ in a clockwise direction, choosing $v_{1}$ arbitrarily, and let $\epsilon_{i}$ be the edge connecting $v_{i}$ and $v_{i+1}$ for $i=1, \ldots, n$, where $v_{n+1}=v_{1}$. We first consider the possible $S$ that do not contain $\epsilon_{n}$ : Since $\epsilon_{n} \notin S$, such $S$ can be understood as a subset of the vertex set of $P_{n}$, and the contribution of such $S$ to $p_{\lambda}$ in the expansion of $X_{C_{n}}$ is the same as the coefficient of $p_{\lambda}$ in the expansion of $X_{P_{n}}$, which is

$$
(-1)^{n-\sum_{i=1}^{n} m_{i}} \frac{\left(\sum_{i=1}^{n} m_{i}\right) !}{\prod_{i=1}^{n}\left(m_{i}\right) !}
$$

We now consider the possible $S$ that do contain $\epsilon_{n}$ : There are cases to consider, depending on the number of vertices $j$, where $j \geqslant 2$, in the connected component of $S$ that contains $\epsilon_{n}$. For each $j$, there are $(j-1)$ possible connected components depending on the smallest labeled vertex of the component, which can be $v_{n-j+2}, \ldots, v_{n}$. After we identify the connected component containing $\epsilon_{n}$ in $C_{n}$, the remainder of the graph is the path graph with $(n-j)$ vertices to which we can apply the third part of this theorem. The overall contribution of such $S$ is thus

$$
\sum_{j=2}^{n}(-1)^{j-1}(j-1)(-1)^{n-j-\left(\sum_{i=1}^{n} m_{i}\right)+1} \frac{\left(\left(\sum_{i=1}^{n} m_{i}\right)-1\right) !\left(m_{j}\right)}{\prod_{i=1}^{n}\left(m_{i}\right) !} .
$$

We now add (2) and (3) to obtain that the coefficient of $p_{\lambda}$ is

$$
(-1)^{n-\sum_{i=1}^{n} m_{i}} \frac{\left(\sum_{i=1}^{n} m_{i}\right) !}{\prod_{i=1}^{n}\left(m_{i}\right) !}\left(1+\sum_{j=2}^{n}(j-1) \frac{m_{j}}{\sum_{i=1}^{n} m_{i}}\right) .
$$

Remark 9. A natural question to ask is whether any of these bases is Schur positive, that is, a positive linear combination of Schur functions. The answer is not at all obvious, since the basis whose generators stem from complete graphs is trivially Schur positive, whereas the basis whose generators stem from star graphs is not as $X_{S_{4}}$ is not. 


\section{Acknowledgements}

The authors would like to thank Boram Park for helpful conversations, the referee for helpful comments, and Ajou University where the research took place.

\section{References}

[1] J. Aliste-Prieto and J. Zamora. Proper caterpillars are distinguished by their chromatic symmetric function. Discrete Math., 31:158-164, 2014.

[2] R. Ehrenborg and S. van Willigenburg. Enumerative properties of Ferrers graphs. Discrete Comput. Geom., 32:481-492, 2004.

[3] V. Gasharov. Incomparability graphs of $(3+1)$-free posets are $s$-positive. Discrete Math., 15:193-197, 1996.

[4] M. Guay-Paquet. A modular relation for the chromatic symmetric functions of $(3+1)-$ free posets. arXiv:1306.2400

[5] J. Martin, M. Morin, and J. Wagner. On distinguishing trees by their chromatic symmetric functions. J. Combin. Theory Ser. A, 115:237-253, 2008.

[6] R. Orellana and G. Scott. Graphs with equal chromatic symmetric function. Discrete Math., 320:1-14, 2014.

[7] R. Stanley. A symmetric function generalization of the chromatic polynomial of a graph. Adv. Math., 111:166-194, 1995.

[8] R. Stanley and J. Stembridge. On immanants of Jacobi-Trudi matrices and permutations with restricted position. J. Combin. Theory Ser. A, 62:261-279, 1993. 